\title{
EVALUACIÓN DE LA REACCIÓN ACROSOMAL INDUCIDA POR EL IONÓFORO DE CALCIO: UNA APROXIMACIÓN MÁS REAL DE LA CAPACIDAD FECUNDANTE DEL ESPERMATOZOIDE.
}

\author{
Wálter Darío Cardona Maya, Martha Olivera Ángel y Ángela Patricia Cadavid.
}

Grupo Reproducción-Biogénesis, Universidad de Antioquia, Medellin, Colombia.

\begin{abstract}
Resumen.- OBJETIVO: Evaluar la reacción acrosomal inducida por el ionóforo de calcio en espermatozoides de un grupo de individuos con fertilidad desconocida.

MÉTODO: Cada individuo donó una muestra de semen la cual fue evaluada para determinar la cantidad de espermatozoides que presentaban reacción acrosomal al estimularlos con el ionóforo de calcio después de la capacitación espermática. Se utilizó la lectina Pisum sativum aglutinina (microscopía de fluorescencia) y el anticuerpo anti-CD46 (citometría de flujo).
\end{abstract}

RESULTADOS: Cinco de los seis individuos presentaron un aumento en el número de espermatozoides reaccionados al estimularlos con ionóforo de calcio comparado con el porcentaje de espermatozoides que presentaron reacción acrosomal espontánea tanto por microscopía de fluorescencia $(7.4+/-2.4$ vs $22.5+/-10.1)$, como por citometría de flujo $(8.5+/-2.8$ vs $25.4+/-6.0)$; en el otro individuo los valores de la reacción acrosomal con el ionóforo de calcio fueron similares a los de la reacción espontánea.

CONCLUSIONES: Ambas técnicas son útiles para evaluar in vitro la capacidad de los espermatozoides para sufrir la reacción acrosomal posterior a la capacitación, sin necesidad de que exista fusión entre el espermatozoide y el oocito, pero la citometría de flujo es mas objetiva y permite visualizar un mayor número de células. De otro lado, aunque el individuo que no respondió al estímulo con el ionóforo de calcio tiene fertilidad desconocida, los resultados obtenidos permiten proponer que la evaluación de la reacción acrosomal podría ser una herramienta útil en el estudio de los hombres que consultan por infertilidad.

Palabras clave: Espermatozoide. Reacción acrosomal. Fertilidad. Ionoforo de calcio.

Summary.- OBJECTIVES: The aim of this investigation was to evaluate the acrosome reaction by fluorescence microscopy and the flow citometry in a group of men with unknown fertility.

METHODS: Each individual gave a semen sample for the analysis of the calcium ionophore-induced acrosome reaction after the capacitation process, using lectin Pisum sativum aglutinina (fluorescence microscopy) and antibody anti-CD46 (flow citometry).

RESULTS: Five of out six individuals increased the number of the sperm with calcium ionophore-induced acrosome reaction versus the percentage of spontaneous acrosome reaction, using fluorescence microscopy $(7.4+/-2.4$ vs 
$22.5+/-10.1)$ and flow citometry $(8.5+/-2.8$ vs $25.4+/-6.0$ ); On the other hand, the other individual the values of the acrosomal reaction with calcium ionophore A23 187 were similar to those of the spontaneous reaction.

CONCLUSIONS: Both techniques are useful to evaluate the in vitro capacity of the spermatozoa to undergo acrosomal reaction, after the capacitation, without fusion between the sperm and the egg, but the flow citometry is more objective and allow the detection of a higher cell numbers of cells. On the other hand, although the individual that did not respond to the stimulus with calcium ionophore has unknown fertility, the obtained results allow to propose that the evaluation of the acrosomal reaction could be a useful tool in the study of the men who consult for infertility.

Keywords: Spermatozoa. Acrosome reaction. Fertility. Calcium ionophore.

\section{INTRODUCCIÓN}

La fecundación es un proceso en el cual se requiere la interacción entre gametos masculinos y femeninos, maduros y competentes, para que éstos se fusionen y formen un nuevo individuo $(1,2)$. El diálogo celular entre ambos gametos ha sido motivo de estudio desde 1919 (3) y aún en la actualidad los detalles de este proceso son poco entendidos.

Cuando el espermatozoide viaja hacia el oocito sufre cambios iniciales que modulan su estado funcional: en organismos de fecundación externa como la estrella de mar y los peces teleosteos los espermatozoides desarrollan su potencial de movilidad sólo después de ser eyaculados; en los organismos de fecundación externa como los reptiles, los pájaros y los mamíferos, los espermatozoides desarrollan el potencial móvil cuando pasan a través del epidídimo $(4,5)$, y es durante este paso, que sufre modificaciones morfológicas y bioquímicas necesarias para convertirse en célula fecundante. Aunque la maquinaria celular involucrada en el desarrollo de estos cambios es heterogénea y requiere de diversos elementos, estudios recientes indican que el flujo de iones de $\mathrm{Ca}^{2++}$ $\mathrm{y} \mathrm{K}^{+}$es clave en el diálogo molecular que se establece entre el espermatozoide, su medio ambiente y el óvulo; es decir, tanto en los procesos de maduración como de capacitación espermática (6-8).

A principios de 1950 J.C. Dan estudiando la penetración de los espermatozoides de estrella de mar en los oocitos, mediante microscopía de contraste de fase y microscopía electrónica, descubrió el proceso de reacción acrosomal $(9,10)$ y ahora está plenamente establecido que dicho proceso es un paso indispensable durante la fertilización en muchas especies, incluyendo el humano. Después de este hallazgo los científicos le dieron importancia al espermatozoide como elemento fundamental en el proceso de fecundación y gracias a ésto, nuevamente J.C. Dan (1 1) descubrió que la reacción acrosomal es dependiente de calcio.

La reacción acrosomal es un proceso especializado de fusión de la membrana citoplasmática con la membrana acrosomal externa en la zona apical de la cabeza espermática originando la liberación de las enzimas almacenadas en esta vesícula y la exposición de la membrana acrosomal interna. La importancia de la reacción acrosomal podría ser la liberación de las enzimas hidrolíticas que son requeridas para que el espermatozoide pueda penetrar la zona pelúcida, además de la expresión de moléculas que actúan como receptores para que pueda ingresar al oocito y ocurra la fusión entre gametos (12, 13). La reacción acrosomal puede ser determinada por varias técnicas: azul de coomasie (14), lectina Pisum sativum aglutinina marcada con isotiocianato de fluoresceína (PSA-FITC) (14), microscopía electrónica (15), citometría de flujo con los anticuerpos antiCD46 (16) ó anti-GB24 (17), entre otras.

La lectina PSA-FITC tiene afinidad por los residuos de manosa y galactosa de las glicoproteínas presentes en la membrana plasmática del dominio acrosomal de la cabeza del espermatozoide, es una tinción negativa que permite determinar la desaparición de la membrana plasmática debido a que durante la reacción acrosomal se desaparece el dominio acrosomal del espermatozoide; De otro lado, el anti-CD-46 unido a un fluorocromo, es una coloración positiva, debido a que la proteína CD46 está localizada unicamente en la membrana interna acrosomal y sólo los espermatozoides que sufren reacción acrosomal completa pueden ser marcados (16).

Es conocido que el número de espermatozoides humanos que presentan reacción acrosomal espontánea in vitro en condiciones capacitantes es bajo y variable, menos del $15 \%$ (18), pero este número puede ser incrementado por el contacto con oocitos $(19,20)$ o con factores inductores de la reacción acrosomal como el fluido folicular (5), el suero sanguíneo (21), la progesterona (22), el ionóforo de calcio A23187 $(23,24)$ y el forbol miristato acetato (24).

Los espermatozoides de algunos hombres con infertilidad inexplicada muestran una disminución en la capacidad de sufrir reacción acrosomal 
(25), lo que podría indicar alteraciones en algún aspecto funcional del proceso de fecundación.

Históricamente, se han realizado muchos intentos para identificar una o varias características del semen que permitan predecir la capacidad de los espermatozoides para fecundar. Los laboratorios de reproducción asistida han usado pruebas como el ensayo de penetración de oocitos de hamster dorado libres de zona pelúcida (26), el cariotipo de espermatozoides (27), la desnaturalización del DNA (28), la evaluación de la membrana espermática (28) y la reacción acrosomal (13), entre otras.

De las anteriores pruebas, la integridad de la membrana y la desnaturalización del DNA, que evalúan la funcionalidad espermática, no presentaron una distribución homogénea en un grupo de individuos fértiles, lo cual deja en entre dicho la capacidad de discriminación de estas pruebas para el diagnóstico de infertilidad (29). El ensayo de penetración de oocitos de hámster dorado libres de zona pelúcida (27), por su parte, es demorado, costoso y sólo permite analizar la capacidad fecundante de un pequeño grupo de espermatozoides del eyaculado y no la capacidad de atravesar la zona pelúcida de éstos.

La determinación de la reacción acrosomal por citometría de flujo es un método alternativo para evaluar la capacidad de los espermatozoides de sufrir la reacción acrosomal in vitro, posterior a la capacitación, sin necesidad de que exista fusión entre el espermatozoides y el oocito; además puede dar información de aproximadamente 10.000 espermatozoides, haciendo esta evaluación más representativa del eyaculado.

El objetivo de este trabajo fue comparar la evaluación de la reacción acrosomal por las técnicas de microscopía de fluorescencia y citometría de flujo en un grupo de individuos.

\section{METODOLOGIAA}

\section{Población de estudio}

Seis individuos aparentemente sanos con los parámetros seminales normales con respecto a la OMS (29). A cada muestra de semen se le determino el porcentaje de reacción acrosomal espontánea, sin inducción y el porcentaje de reacción acrosomal inducida inducida por el ionóforo de calcio.

\section{Muestras de semen}

Cada individuo donó una muestra de semen que fue tomada en un recipiente estéril mediante mas- turbación, después a una abstinencia sexual de 2 a 5 días. Uno de los individuos, que se describirá mas adelante, donó tres muestras de semen.

\section{Separación y capacitación espermática}

Simultáneamente se realizó la separación y la capacitación espermática variando el protocolo descrito por Jondet (30) con incubación de 5 horas en medio HAM F-12 suplementado con $35 \mathrm{mg} / \mathrm{ml}$ de BSA (Sigma Chemical Company, St Lovis, MO, USA), lo que permitía obtener espermatozoides móviles y capacitados.

\section{Inducción de la reacción acrosomal}

La inducción de la reacción acrosomal se realizó con 10 uM de ionóforo de calcio A23187 (Sigma Chemical Company, St Louis, MO, USA) durante 3 horas a $37^{\circ} \mathrm{C} / 5 \% \mathrm{CO}_{2}$.

\section{Evaluación de la reacción acrosomal por microscopía de fluorescencia}

Aproximadamente $1 \times 10^{6}$ espermatozoides fueron lavados dos veces en buffer fosfato salino (PBS), depositados sobre portaobjetos, fijados por exposición a metanol frío/30 segundos e incubadas con la lectina Pisum Sativum (Sigma Chemical Company, St Loui, MO, USA) a una concentración de 50

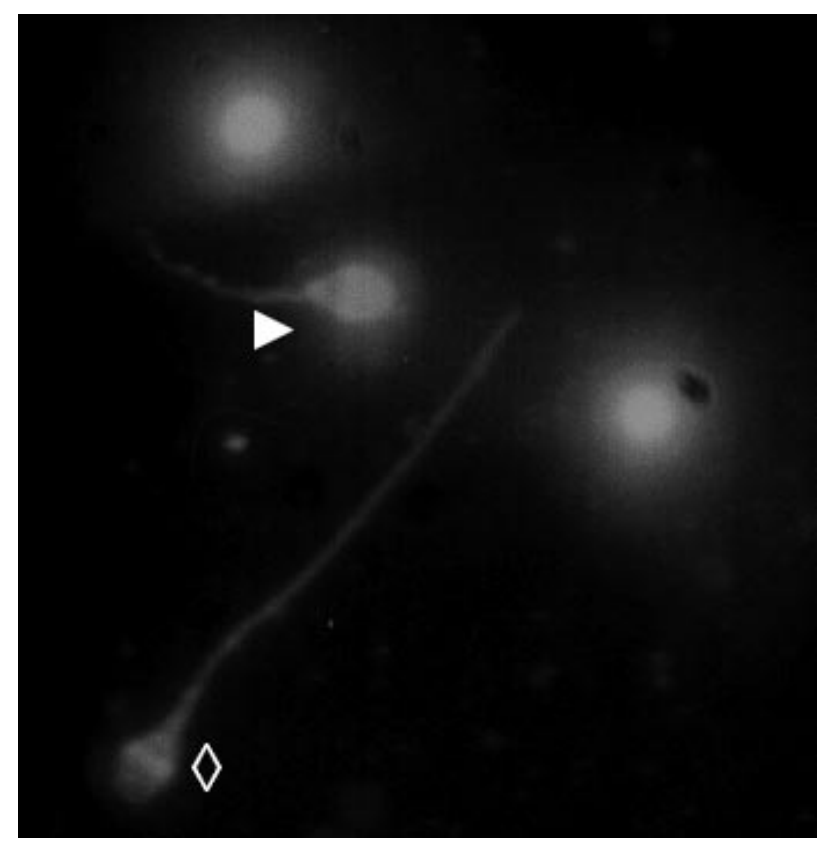

FIGURA 1. Patrón de espermatozoides coloreados con la lectina PSA marcada con iosotiocianato de fluoresceína, espermatozoides con $(\nabla)$ y $\sin (\downarrow)$ reacción acrosomal. 
$\mu \mathrm{g} / \mathrm{ml}$ en PBS durante 30 minutos/ cámara húmeda. Luego de dos lavados con PBS, los extendidos secos fueron montados en glicerol al $90 \%$ en PBS y se evaluaron 100 espermatozoides en el microscopio de fluorescencia con un aumento de 100X, para determinar el porcentaje de espermatozoides reaccionado.

Se consideraron como espermatozoides reaccionados aquellos que presentaban fluorescencia solo en la región ecuatorial y espermatozoides intactos, aquellos que presentaron todo el acrosoma fluorescente, los espermatozoides morfológicamente alterados no fueron incluidos en el recuento para minimizar el número de espermatozoides falsos positivos. (Figura 1).

\section{Evaluación de la reacción acrosomal por citometría de flujo}

Aproximadamente $1 \times 10^{6}$ espermatozoides fueron incubados durante 30 minutos a temperatura ambiente con Ig $G$ humana (Miltenyu Bistec $\mathrm{GMBH}$, Bergisch Gladbach, Germany) para bloquear de la unión a los receptores Fc. Después de 2 lavados con PBS a $250 \mathrm{~g} / 8$ minutos se agregaron $10 \mu \mathrm{l}$ de anticuerpo $\lg G$ de ratón contra CD46 humano conjugado

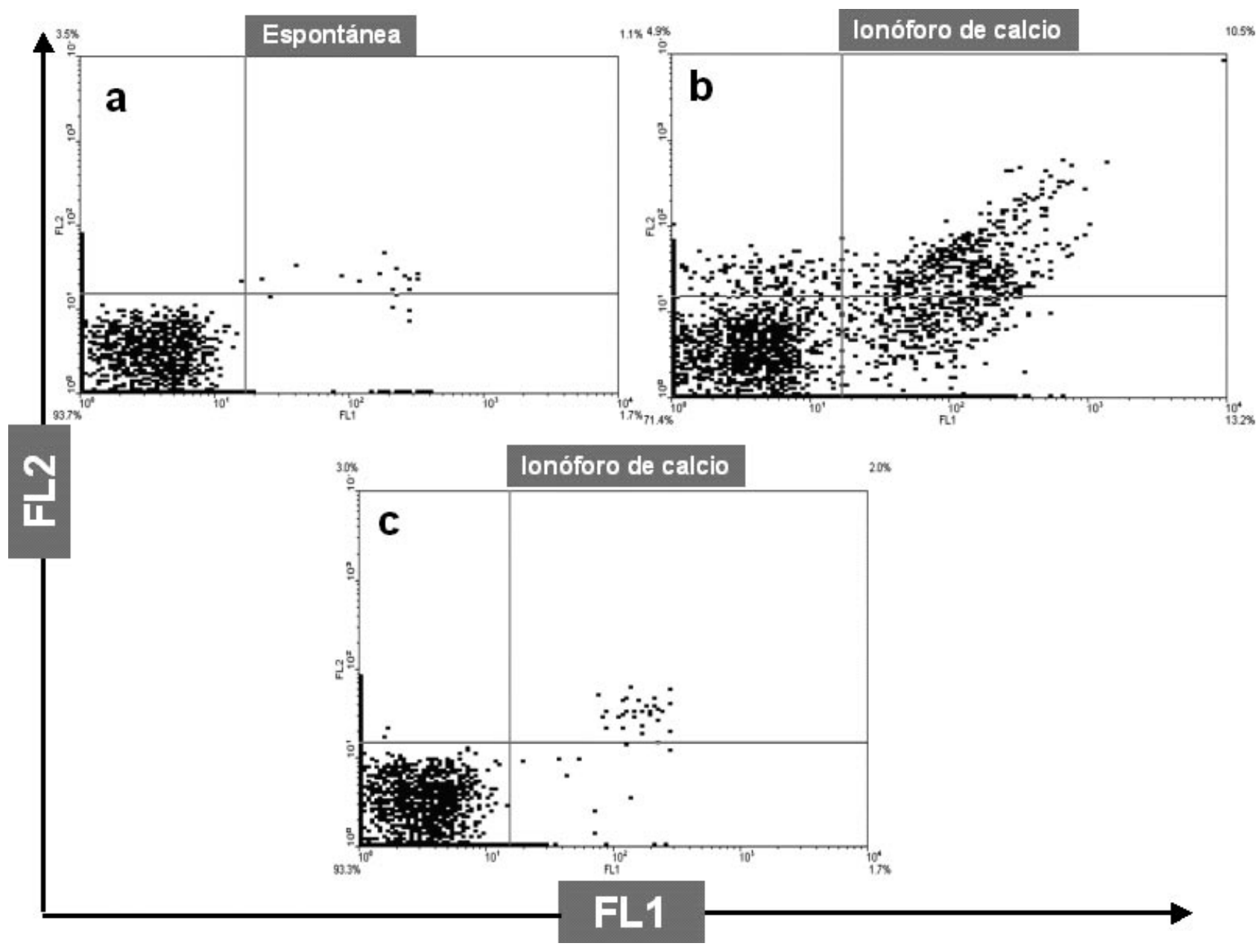

FIGURA 2. Resultados de citometría de flujo. Diagrama de puntos de espermatozoides marcados con anticuerpo anti CD46-FITC y ioduro de propidio. Cada panel está dividido en cuatro cuadrantes, representando diferentes subpoblaciones de espermatozoides acorde con su estado acrosomal y viabilidad: el cuadrante superior izquierdo representa los espermatozoides permeables al ioduro (células muertas) y con acrosoma intacto, el cuadrante superior derecho representa los espermatozoides permeables al yoduro pero que han sufrido reacción acrosomal, en el cuadrante inferior izquierdo se localizan las células vivas y con acrosoma intacto y en el cuadrante inferior derecho se ubican los espermatozoides vivos reaccionados. (a) reacción acrosomal espontánea, (b) ensayo representativo de uno de los individuos que responde a la inducción con ionóforo de calcio A23 187 y (c) individuo que no respondió a la inducción de la reacción acrosomal mediante el estímulo con ionóforo de calcio. 
con FITC (BD PharMingen San Diego, USA); en otra alícuota se agregó un control de isotipo utilizando un anticuerpo lgG de ratón contra trinitrofenol (BD PharMingen San Diego, USA). Las muestras se incubaron a $\mathrm{T}^{\circ}$ ambiente protegidas de la luz, se lavaron con PBS y luego se les adicionó $2.5 \mu \mathrm{g} / \mathrm{ml}$ de ioduro de propidio (Sigma Chemical Company, St Louis, MO, USA) para evaluar la viabilidad espermática (31).

\section{Análisis estadístico}

La comparación estadística entre los grupos de reacción acrosomal espontánea e inducida, fue realizada mediante la prueba de Mann-Whitney; el análisis de concordancia entre los valores de espermatozoides reaccionados, obtenidos mediante microscopía de fluorescencia y citometría de flujo se realizó por la prueba de Blant Altman. El paquete estadístico utilizado fue Prism ${ }^{\circledR}$ version 4.0.

\section{RESULTADOS}

En la Figura 2 se ilustran resultados representativos obtenidos mediante citometría de flujo, en FL1 las células marcadas con el anticuerpo antiCD46 FITC y en FL2 las células que adquirieron o no el yoduro de propidio. La Figura 2a representa la reacción acrosomal espontánea y la Figura $2 b$ la reacción acrosomal inducida por el ionóforo de calcio que fue mayor que la reacción espontánea, 8.5 +/- 2.8 vs $25.4+/-6$, respectivamente. Uno de los individuos (el \#1) no respondió al estímulo con el io-
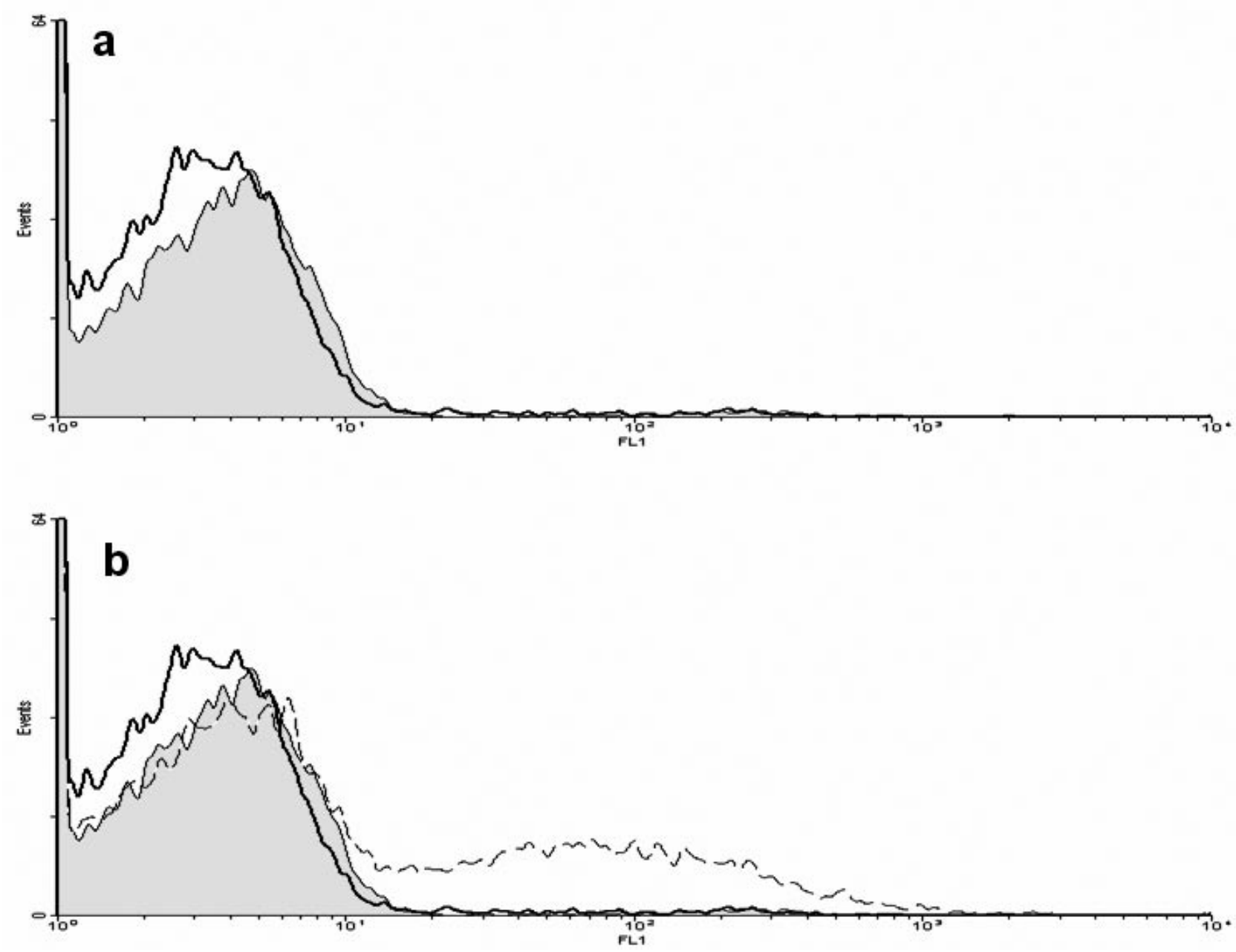

FIGURA 3. Intensidad de fluorescencia media, comparando entre un individuo control y el individuo que no respondió al estímulo por calcio. Histograma representativo de los ensayos realizados donde se observa la diferencia entre la reacción acrosomal espontánea (a, sombra), y la reacción acrosomal inducida mediante ionóforo de calcio en el individuo motivo de estudio (a, línea continua) y un individuo que sí responde a calcio (b, línea punteada). 
nóforo de calcio (Figura 2c); se repitió la muestra tres veces con resultados similares.

Para confirmar los resultados obtenidos, se determinó la intensidad de fluorescencia media y se observó que el individuo que no respondía al estímulo con ionóforo de calcio (línea continua Figura $3 a$ y $3 b)$ presentaba una intensidad similar a la obtenida en la reacción acrosomal espontánea (sombra Figura 3) y menor que la intensidad de un individuo control (línea punteada Figura 3b).

En la evaluación de la reacción acrosomal con la lectina PSA-FITC mediante microscopía de fluorescencia se observaron resultados similares a los anteriores, es decir se encontró la misma respuesta del individuo \# 1 que no respondió al estímulo con ionóforo de calcio (Figura 4).

El individuo que no respondió al estímulo con ionóforo de calcio, es aparentemente sano, con una edad de 32 años, con fertilidad desconocida y el cual, como se observa en la Tabla I, presenta en las tres muestras de semen todos sus parámetros seminales normales según los estándares de la Organización Mundial de la Salud (40).
Después de analizar los datos obtenidos en las muestras de los 5 individuos y en tres ensayos del individuo \# 1 que no respondió al estimulo con ionóforo de calcio, los resultados muestran que a diferencia de los 5 individuos donde se observó un aumento estadísticamente significativo en el porcentaje de espermatozoides reaccionados al estimular con ionóforo de calcio $(p<0.01)$ el individuo \# 1 no presentó este aumento $(p>0.05)$, ni con la lectina PSAFITC ni con el anticuerpo anti-CD46-FITC, (Figura 4a y 4 b) respectivamente. El análisis de concordancia entre ambos métodos, microscopía de fluorescencia y citometría de flujo, teniendo en cuenta los valores obtenidos en las 8 muestras analizadas, mostró una alta concordancia, (Figura 5).

\section{DISCUSIÓN}

La evaluación de la reacción acrosomal podría ser una herramienta útil en el estudio de la infertilidad. Liu DY y cols (32) muestran que la reacción acrosomal inducida por el ionóforo de calcio A23187 tiene una correlación positiva $(p<0.05)$ con los valores de fertilización en pacientes con semen teratozoospérmico (morfología normal $<15 \%$ ), lo
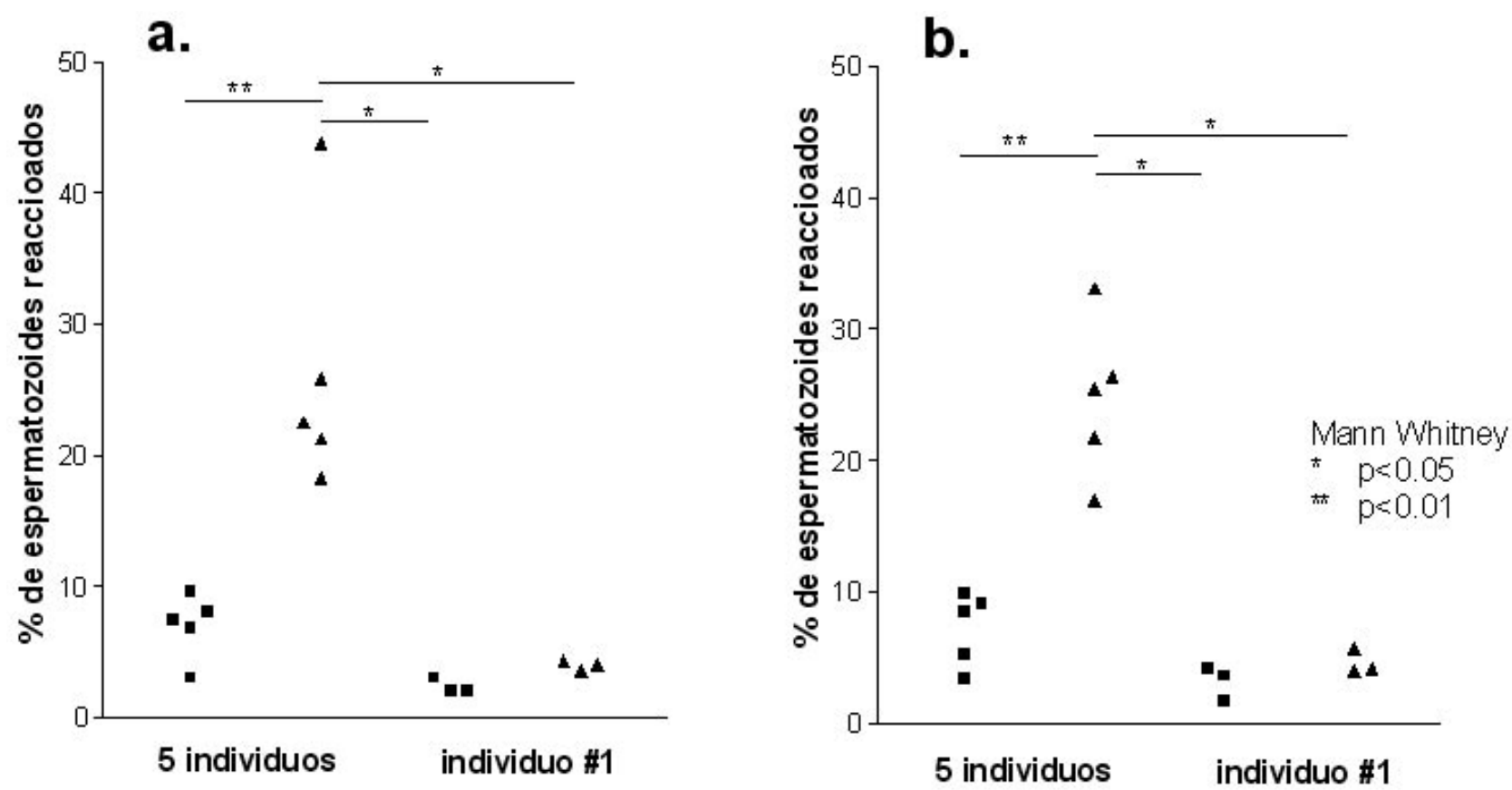

FIGURA 4. Comparación entre reacción acrosomal espontánea e inducida con ionóforo de calcio A23 187. Evaluación de la reacción acrosomal inducida por el ionóforo de calcio A23 187 después del proceso de capacitación durante 5 horas en medio HAM F-12 suplementado con $35 \mathrm{mg} / \mathrm{ml}$ de albúmina sérica bovina. El porcentaje de espermatozoides que sufrieron reacción acrosomal fue evaluado mediante PSA-FITC por (a) microscopía de fluorescencia y (b) el anticuerpo anti-CD46 usando citometría de flujo. Se muestran los resultados comparando los cinco individuos estudiados con el individuo \# 1 que no respondió al estímulo con ionóforo de calcio. 
TABLA I. Características seminales de los individuos pre y post capacitación Descripción de los parámetros seminales analizados de cada uno de los individuos, comparados frente al valor de referencia de la Organización Mundial de la Salud de 1999 (29); además se muestran los resultados de movilidad y concentración recuperada después de realizar la capacitación de cada una de las muestras. El individuo \# 1 sombreado es el que no respondió al estímulo con el ionóforo de calcio.

\begin{tabular}{|c|c|c|c|c|c|c|c|}
\hline & $\begin{array}{c}\text { Individuo } \\
\qquad \begin{array}{l}\# \\
(n=3)\end{array}\end{array}$ & $\begin{array}{c}\# 2 \\
(n=1)\end{array}$ & $\begin{array}{c}\# 3 \\
(n=1)\end{array}$ & $\begin{array}{c}\# 4 \\
(n=1)\end{array}$ & $\begin{array}{c}\# 5 \\
(n=1)\end{array}$ & $\begin{array}{c}\# 6 \\
(n=1)\end{array}$ & Valor OMS \\
\hline Volumen (ml) & $\begin{array}{c}2.6 \\
(2.4-2.8)\end{array}$ & 4.2 & 3.2 & 5 & 3.2 & 2.8 & $\geq 2.0$ \\
\hline Viabilidad & $\begin{array}{c}85 \\
(84-86) \\
\end{array}$ & 85 & 86 & 80 & 88 & 83 & $>50$ \\
\hline Morfología & (> a $15 \%)$ & \multicolumn{6}{|c|}{$>15 \%$} \\
\hline $\begin{array}{l}\text { Concentración pre-capacitación } \\
(106 / \mathrm{ml})\end{array}$ & $\begin{array}{c}60.5 \\
(56-65)\end{array}$ & 60 & 34 & 75 & 35 & 41 & $\geq 20$ \\
\hline $\begin{array}{l}\text { Concentración post-capacitación } \\
(106 / \mathrm{ml})\end{array}$ & $\begin{array}{c}28 \\
(28-30) \\
\end{array}$ & 34 & 12 & $34^{\circ}$ & 16 & 19 & $\mathrm{np}$ \\
\hline $\begin{array}{l}\text { Movilidad a+b pre-capacitación } \\
(\%)\end{array}$ & $\begin{array}{c}60 \\
(55-65) \\
\end{array}$ & 60 & 55 & 60 & 65 & 60 & $>50$ \\
\hline $\begin{array}{l}\text { Movilidad a+b post-capacitación } \\
(\%)\end{array}$ & $\begin{array}{c}86.6 \\
(82-90)\end{array}$ & 82 & 90 & 85 & 90 & 86 & $\mathrm{np}$ \\
\hline
\end{tabular}

\section{Concordancia entre microscopía de fluorescencia y citometría de flujo}

\section{Bland-Altman}

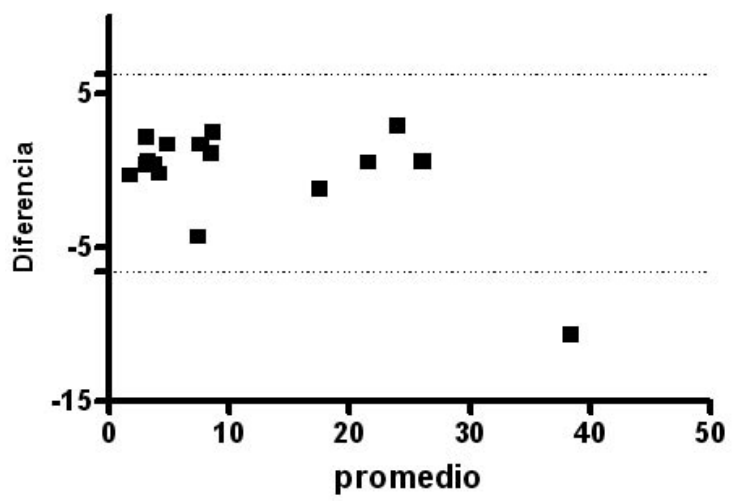

FIGURA 5. Concordancia entre los valores de reacción acrosomal entre microscopía de fluorescencia y citometría de flujo, tanto espontánea como inducida con ionóforo de calcio $(n=16)$ cual evidencia que la reacción acrosomal inducida por el ionóforo puede generar información adicional de predicción de las tasas de fertilización, como lo sugieren también otros autores $(33,34)$, aunque el mecanismo sigue siendo desconocido. Cummins J.M. y cols. en 1991 (33) clasificaron a los individuos con menos de $5 \%$ de reacción acrosomal inducida por el ionóforo de calcio como pacientes con problemas en los procedimientos de fertilización in vitro y sugieren que a éstos se les realicen otros procedimientos como aumento en la concentración espermática para la inseminación o el análisis de dos muestras tomadas con un intervalo de 1 hora con el fin de aumentar el número de espermatozoides que se utilizarán.

Otros estudios del grupo de Liu D.Y. y cols. $(35,36)$, muestran que individuos infértiles con desórdenes en la inducción de la reacción acrosomal y con bajos índices de fertilización in vitro tenían un promedio de $6 \%$ (con un rango de 0 a 16), de inducción de la reacción acrosomal estimulada con oocitos. En forma similar, hombres normozoospérmicos con me- 
nos del $16 \%$ de reacción acrosomal tenían menos del $20 \%$ de penetración a zona pelúcida y de éstos los que tenían menos del $10 \%$ de reacción no penetraron la zona pelúcida (36). Los anteriores resultados son también confirmados por los hallazgos de Esterhuzen $A D$ y cols (2) quienes encuentran que pacientes con espermogramas normales pero con menos del 15\% de inducción de la reacción acrosomal por zona pelúcida solubilizada tenían malos resultados en los procedimientos de fecundación in vitro. Estos hallazgos indican que existen desórdenes espermáticos que no se pueden evaluar con las metodologías empleadas de rutina en los laboratorios de espermiología.

Algunos investigadores, reportan que la progesterona (16) y los oligosacáridos como la manosa y la N-acetilglucosamina (37) inducen reacción acrosomal parcial pero no reacción acrosomal completa; ésto lo pueden concluir gracias a que los trabajos son realizados con microscopía de fluorescencia usando la PSA-FITC y con citometría de flujo con el anticuerpo anti-CD46 y ambos trabajos concluyen que el ionóforo de calcio A23187 es un inductor de reacción acrosomal completa, por lo cual se puede evaluar por cualquiera de las dos técnicas $(16,37)$. El término reacción acrosomal parcial y completa hace referencia más al estado acrosomal de los espermatozoides de un individuo que al número de células reaccionadas; la reacción acrosomal parcial se refiere a la liberación del contenido acrosomal o pérdida de la membrana acrosomal externa y la reacción acrosomal completa se refiere a la exposición de la membrana acrosomal interna y a la pérdida completa del contenido acrosomal $(15,38)$. Como se mencionó anteriormente, la PSA evalúa el contenido acrosomal - la membrana acrosomal externa por desaparición de ésta, a diferencia del anti-CD46 el cual permite detectar el paso final de la reacción acrosomal por la exposición de la membrana acrosomal interna.

El ionóforo de calcio es un buen inductor de la reacción acrosomal debido a que provoca un aumento de flujo de calcio hacia el espacio intracelular el cual se asocia con la salida de iones $\mathrm{H}+$ y por lo tanto un aumento del $\mathrm{pH}$. Además el aumento de la concentración interna de calcio genera la liberación de los almacenamientos de calcio interno provocando un incremento sustancial de la concentración de calcio citosólico que origina la activación de diferentes vías de señalización lo cual finalmente permite que las membranas se fusionen $(39,40)$.

Los resultados de nuestro estudio sugieren que las dos técnicas usadas para evaluar la reacción acrosomal inducida por ionóforo del calcio, microscopía de fluorescencia y citometría de flujo, son muy similares y nos permitió identificar un individuo que no respondía a este estímulo. La citometría de flujo tiene la ventaja de ser más objetiva en la evaluación y diferenciar las células vivas de las muertas. Los ensayos con la PSA-FITC no incluyeron el uso del colorante vital como lo describen otros autores (14), aunque los espermatozoides alterados morfológicamente no fueron incluidos en el recuento para minimizar la inclusión de espermatozoides falsos positivos; además las muestras de semen usadas con cada una de las técnicas siempre tenían altas proporciones de células móviles (>80\%).

La evaluación de la reacción acrosomal mediante citometría de flujo es una prueba que permite evaluar un aspecto funcional del espermatozoide y se puede hacer en poco tiempo, por lo tanto proponemos que en los programas de reproducción asistida se realice, como una técnica de rutina, la determinación de la reacción acrosomal mediante citometría de flujo con el anticuerpo anti-CD46, simultáneamente con el espermograma. Esto permitiría obtener un beneficio económico para los pacientes que podrían evitar gastos en pruebas que no brindarán una solución al problema; por ejemplo, en un individuo que el ionóforo de calcio no induzca la reacción acrosomal, no se realizarían inseminaciones artificiales y más bien el procedimiento elegido en este caso sería la inyección intracitoplasmática del espermatozoide.

\section{BIBLIOGRAFÍA Y LECTURAS RECOMENDADAS (*lectura de interés $y$ ** lectura fundamental)}

**1. CARDONA MAYA, W.D.; CADAVID JARAMILLO, A.P.: "Complementariedad intergamentos: breve revisión”. Arch. Esp. Urol., 57: 1107, 2004.

2. ESTERHUIZEN, A.D.; FRANKEN, D.R.; LOURENS, J.G. y cols.: "Clinical importance of zona pellucida-induced acrosome reaction and its predictive value for IVF". Hum. Reprod., 16: 138, 2001.

3. LILLIE, F.: "Problems of Fertilization". Chicago IL: University of Chicago Press, 1919.

4. BALDI, E.; LUCONI, M.; BONACCORSI, L. y cols.: "Intracellular events and signaling pathways involved in sperm acquisition of fertilizing capacity and acrosome reaction". Front Biosci, 5: 110, 2000.

5. MORTIMER, D.; CAMENZIND, A.R.: "The role of follicular fluid in inducing the acrosome reaction of human spermatozoa incubated in vitro". Hum. Reprod., 4: 169, 1989. 
6. ARNOULT, C.; KAZAM, I.G.; VISCONTI, P.E. y cols.: "Control of the low voltage-activated calcium channel of mouse sperm by egg ZP3 and by membrane hyperpolarization during capacitation". Proc. Natl. Acad. Sci. USA, 96: 6757, 1999.

7. MUÑOZ-GARAY, C.; DE LA VEGA -BELTRAN, J.L.; DELGADO, R. y cols.: "Inwardly rectifying $\mathrm{K}(+)$ channels in spermatogenic cells: functional expression and implication in sperm capacitation”. Dev. Biol., 234: 261, 2001.

8. ZENG, Y.; CLARK, E.N.; FLORMAN, H.M.: "Sperm membrane potential: hyperpolarization during capacitation regulates zona pellucida-dependent acrosomal secretion". Dev. Biol., 171: 554, 1995.

9. DAN, J.C.: "Reaction to egg-water and other stimuli". Biol. Bull., 103: 54, 1952.

10. DAN, J.C.: "Sperm entrance in echinoderms; observed with the phase contrast microscope". Biol. Bull., 99: 399, 1950.

11. DAN, J.C.: "Effect of $\mathrm{Ca} 2++$ deficiency". Biol. Bull., 107: 335, 1954.

12. MYLES, D.G.: "Molecular mechanisms of spermegg membrane binding and fusion in mammals". Develop. Biol., 158: 35, 1993.

13. SNELL, W.J.; WHITE, J.M.: "The molecules of mammalian fertilization". Cell, 85: 629, 1996.

14. CROSS, N.; MORALES, P.; OVERSTREET, J. y cols.: "Two simple methods for detecting acrosome reacted human sperm". Gamete Res., 15: 213, 1986.

*15. KOHN, F.M.; MACK, S.R.; SCHILL, W.B. y cols.: "Detection of human sperm acrosome reaction: comparison between methods using double staining, Pisum sativum agglutinin, concanavalin A and transmission electron microscopy". Hum. Reprod., 12: 714, 1997.

*16. JAISWAL, B.S.; EISENBACH, M.; TUR-KASPA, I.: "Detection of partial and complete acrosome reaction in human spermatozoa: which inducers and probes to use?". Mol. Hum. Reprod., 5: 214, 1999.

17. FIERRO, R.; FOLIGUET, B.; GRIGNON, G. y cols.: "Lectin-binding sites on human sperm during acrosome reaction: modifications judged by electron microscopy/flow cytometry". Arch. Androl., 36: 187, 1996.

18. BYRD, W.; WOLF, D.P.: "Acrosomal status in fresh and capacitated human ejaculated sperm". Biol. Reprod., 34: 859, 1986.

19. FRANKEN, D.R.; BASTIAAN, H.S.; KIDSON, A. y cols.: "Zona pellucida mediated acrosome reaction and sperm morphology". Andrologia, 29: 311, 1997.

20. STOCK, C.E.; FRASER, L.R.: "Divalent cations, capacitation and the acrosome reaction in human spermatozoa”. J. Reprod. Fertil., 87: 463, 1989.
**21. YANAGIMACHI, R.: "Fertilization in Mammalian". Knobil E NJ, ed. The Physiology of Reproduction. New York: Raven Press Ltd., 189-318, 1994.

22. PATRAT, C.; SERRES, C.; JOUANNET, P.: "The acrosome reaction in human spermatozoa". Biol. Cell, 92: 255, 2000.

23. JAMIL, K.; WHITE, I.G.: "Induction of acrosomal reaction in sperm with ionophore A23187 and calcium". Arch. Androl., 7: 283, 1981.

24. MORTIMER, S.T.; SWAN, M.A.; MORTIMER, D.: "Fractal analysis of capacitating human spermatozoa". Hum. Reprod., 11: 1049, 1996.

25. CALVO, L.; VANTMAN, D.; BANKS, S.M. y cols.: "Follicular fluid-induced acrosome reaction distinguishes a subgroup of men with unexplained infertility not identified by semen analysis". Fertil Steril, 52: 1048, 1989.

26. YANAGIMACHI, R.; YANAGIMACHI, H.; ROGERS, B.J.: "The use of zona-free animal ova as a test-system for the assessment of the fertilizing capacity of human spermatozoa". Biol. Reprod., 15: 471, 1976.

27. RUDAK, E.; JACOBS, P.A.; YANAGIMACHI, R.: "Direct analysis of the chromosome constitution of human spermatozoa". Nature, 274: 911, 1978.

28. CARDONA-MAYA, W.; BERDUGO, J.; DE LOS RIOS, J. y cols.: "Sperm functional evaluation in fertile men”. Preparación, 2005.

29. WHO.: "WHO Laboratory Manual for the Examination of Human Semen and Sperm-Cervical Mucus Interaction”. Cambridge: Cambridge University Press, 1999.

30. TEA, N.T.; JONDET, M.; SCHOLLER, R.: "Migration-gravity sedimentation: method for collecting motile spermatozoa from human semen". Harrison R, Bonnar J, Thompson W, eds. In Vitro Fertilization, Embryo Transfer and Early Pregnancy: Lancaster: MTP Press Ltd, 117-120, 1984.

31. TAO, J.; CRITSER, E.S.; CRITSER, J.K.: "Evaluation of mouse sperm acrosomal status and viability by flow cytometry". Mol. Reprod. Dev., 36: 183, 1993.

32. LIU, D.Y.; BAKER, H.W.: "Calcium ionophoreinduced acrosome reaction correlates with fertilization rates in vitro in patients with teratozoospermic semen". Hum. Reprod., 13: 905, 1998.

33. CUMMINS, J.M.; PEMBER, S.M.; JEQUIER, A.M. y cols.: "A test of the human sperm acrosome reaction following ionophore challenge. Relationship to fertility and other seminal parameters". J. Androl., 12: 98, 1991.

34. YOVICH, J.M.; EDIRISINGHE, W.R.; YOVICH, J.L.: "Use of the acrosome reaction to ionophore challenge test in managing patients in an assisted reproduction program: a prospective, do- 
uble-blind, randomized controlled study". Fertil Steril, 61: 902, 1994.

35. LIU, D.Y.; BAKER, H.W.: "Disordered acrosome reaction of spermatozoa bound to the zona pellucida: a newly discovered sperm defect causing infertility with reduced sperm-zona pellucida penetration and reduced fertilization in vitro". Hum. Reprod, 9: 1694, 1994.

36 LIU, D.Y.; CLARKE, G.N.; MARTIC, M. y cols.: "Frequency of disordered zona pellucida (ZP)induced acrosome reaction in infertile men with normal semen analysis and normal spermatozoaZP binding”. Hum. Reprod, 16: 1185, 2001.

37. CARDONA-MAYA, W.; CADAVID, A.: "Evaluación de la reacción acrosomal en espermatozoides humanos inducida por los monosacaridos manosa y n-acetilglucosamina". Actas Urol. Esp. 29:676, 2005. 2005.

**38. JAISWAL, B.S.; COHEN-DAYAG, A.; TURKASPA, I. y cols.: "Sperm capacitation is, after all, a prerequisite for both partial and complete acrosome reaction". FEBS Lett., 427: 309, 1998.

39. BREITBART, H.: "Role and regulation of intracellular calcium in acrosomal exocytosis". J. Reprod. Immunol., 53: 151, 2002.

40. BREITBART, H.: "Intracellular calcium regulation in sperm capacitation and acrosomal reaction”. Mol. Cell Endocrinol., 187: 139, 2002. 